\title{
The $\left(Z_{2}\right)^{3}$ symmetry of the non-tri-bimaximal pattern for the neutrino mass matrix
}

\author{
E. I. Lashin ${ }^{1,2,3 *}$, M. Abbas ${ }^{1,2} \nmid$ N. Chamoun ${ }^{4,5 \ddagger}$ and S. Nasri ${ }^{6} \S$, \\ ${ }^{1}$ Ain Shams University, Faculty of Science, Cairo 11566, Egypt. \\ ${ }^{2}$ Centre for Theoretical Physics, Zewail City of Science and Technology, \\ Sheikh Zayed, 6 October City, 12588, Giza, Egypt. \\ 3 The Abdus Salam ICTP, P.O. Box 586, 34100 Trieste, Italy. \\ ${ }^{4}$ Physics Department, HIAST, P.O.Box 31983, Damascus, Syria. \\ ${ }^{5}$ Physikalisches Institut der Universität Bonn, Nußalle 12, D-53115 Bonn, Germany. \\ ${ }^{6}$ Department of Physics, UAE University, P.O.Box 17551, Al-Ain, United Arab Emirates.
}

January 24, 2021

\begin{abstract}
In view of the recent neutrino oscillation data pointing to a non-vanishing value for the smallest mixing angle $\left(\theta_{z}\right)$, we derive and find explicit realizations of the $\left(Z_{2}\right)^{3}$ flavor symmetry which characterizes, for the neutrino mass matrix, uniquely a variant of the tripartite form, originally conceived to lead to the tri-bimaximal mixing with $\theta_{z}=0$, so that to allow now for a non-tri-bimaximal pattern with non-zero $\theta_{z}$. We impose this flavor symmetry in a setting including the charged leptons and we see that it can make room, through higher order terms involving new SM-singlet scalars, for the mass hierarchy of charged leptons. Moreover, within type-I seesaw mechanism augmented with the flavor symmetry, certain patterns occurring in both the Dirac and the Majorana neutrino mass matrices can accommodate all types of mass hierarchies in the effective neutrino mass matrix, but no lepton/baryon asymmetry can be generated. Finally, we discuss how type-II seesaw mechanism, when supplemented with the flavor symmetry, could be used to interpret the observed baryon asymmetry through leptogenesis.
\end{abstract}

Keywords: Neutrino Physics; Flavor Symmetry; Matter-anti-matter.

PACS numbers: 14.60.Pq; 11.30.Hv; 98.80.Cq

\section{Introduction}

Neutrino oscillation experiments have established that neutrino flavor and mass states do mix [1]. The three flavor states $\left(\nu_{e}, \nu_{\mu}, \nu_{\tau}\right)$ are quantum linear combinations of the three light mass states $\left(\nu_{1}, \nu_{2}, \nu_{3}\right)$ through a unitary mixing matrix $U_{L}^{\nu}$ parameterized by three rotation angles $\left(\theta_{x}, \theta_{y}, \theta_{z}\right)$ and three phases $[\delta$ (Dirac phase), $\rho$ and $\sigma$ (Majorana phases $)]$ as follows:

$$
\begin{aligned}
M_{\nu} & =U_{L}^{\nu} M_{\nu}^{\operatorname{diag}}\left(U_{L}^{\nu}\right)^{T} \\
M_{\nu}^{\operatorname{diag}} & =\operatorname{diag}\left(m_{1}, m_{2}, m_{3}\right), \\
U_{L}^{\nu} & =\left(\begin{array}{ccc}
c_{x} c_{z} & s_{x} c_{z} & s_{z} \\
-c_{x} s_{y} s_{z}-s_{x} c_{y} e^{-i \delta} & -s_{x} s_{y} s_{z}+c_{x} c_{y} e^{-i \delta} & s_{y} c_{z} \\
-c_{x} c_{y} s_{z}+s_{x} s_{y} e^{-i \delta} & -s_{x} c_{y} s_{z}-c_{x} s_{y} e^{-i \delta} & c_{y} c_{z}
\end{array}\right) \operatorname{diag}\left(e^{i \rho}, e^{i \sigma}, 1\right) .
\end{aligned}
$$

*elashin@ictp.it

${ }^{\dagger}$ mabbas1978@yahoo.com

${ }_{\ddagger}^{\ddagger}$ nchamoun@th.physik.uni-bonn.de

$\S_{\text {snasri@uaeu.ac.ae }}$ 
where $M_{\nu}$ is the effective neutrino mass matrix which is symmetric assuming the neutrinos are of Majorana type, $m_{i}$ 's are the masses of the neutrino mass states, and with $s_{x} \equiv \sin \theta_{x}, c_{y} \equiv \cos \theta_{y}$ and so on.

Our convention for parameterizing the mixing matrix in Eq. (1) is related to the standard convention used, say, in the recent data analysis of [2], in that the mixing angles coincide in the two conventions but with different nomenclatures; strictly speaking,

$$
\theta_{x} \equiv \theta_{12}, \quad \theta_{y} \equiv \theta_{23}, \quad \theta_{z} \equiv \theta_{13} .
$$

As to the phases, one needs to decompose the unitary mixing matrix $U_{L}^{\nu}$ as

$$
U_{L}^{\nu}=R_{23}\left(\theta_{y}\right) \cdot R_{z}(\delta) \cdot R_{12}\left(\theta_{x}\right) \cdot P
$$

where $R_{12}$ and $R_{23}$ represent rotations around the $z$ and $x$ axes respectively, whereas $R_{z}(\delta)$ and $P$ depend on the convention adopted. The standard parametrization, denoted by a superscript tilde, consists of taking

$$
\widetilde{R_{z}}(\delta)=\left(\begin{array}{ccc}
c_{z} & 0 & s_{z} e^{-i \delta} \\
0 & 1 & 0 \\
-s_{z} e^{i \delta} & 0 & c_{z}
\end{array}\right) \quad, \quad \widetilde{P}=\operatorname{diag}\left(1, e^{i \frac{\phi_{2}}{2}}, e^{i \frac{\left(\phi_{3}+2 \delta\right)}{2}}\right) .
$$

where $\phi_{2}$ and $\phi_{3}$ designate the Majorana phases. On the other hand, in the parametrization we adopt, which has the advantage that the Dirac phase $\delta$ deos not appear in the effective mass term of the neutrinoless double beta decay [3], we have

$$
R_{z}(\delta)=\left(\begin{array}{ccc}
c_{z} & 0 & s_{z} \\
0 & e^{-i \delta} & 0 \\
-s_{z} & 0 & c_{z}
\end{array}\right), \quad, \quad P=\operatorname{diag}\left(e^{i \rho}, e^{i \sigma}, 1\right) .
$$

One can show, by calculating some rephasing invariant quantities [4], that the Dirac phase is the same for both parameterizations while the Majorana phases in the two parameterizations are related through simple linear relations:

$$
\rho=-\frac{\phi_{3}}{2}, \quad \sigma=\frac{\phi_{2}-\phi_{3}}{2} .
$$

Likewise, one can diagonalize the charged lepton mass matrix linking the left handed (LH) leptons to their right handed $(\mathrm{RH})$ counterparts, and thus being not necessarily symmetric, albeit now by a bi-unitary transformation:

$$
M_{l}=U_{L}^{l} \operatorname{diag}\left(m_{e}, m_{\mu}, m_{\tau}\right)\left(U_{R}^{l}\right)^{\dagger} .
$$

The observed neutrino mixing matrix comes from the mismatch between $U_{L}^{l}$ and $U_{L}^{\nu}$ in that one can put experimental constraints on the elements of the flavor mixing matrix $V=\left(U_{L}^{l}\right)^{\dagger} U_{L}^{\nu}$. If one works in the flavor basis where $M_{l} M_{l}^{\dagger}$ is diagonal, then $U_{L}^{l}=\mathbf{1}$ is the identity matrix, and the whole measured flavor mixing matrix originates from $U_{L}^{\nu}$.

The authors of [5] noticed that, dropping the phases on which no definite experimental measurements exist up till now, the experimental constraints impose a specific pattern named tri-bimaximal (TB):

$$
U_{L}^{\nu} \simeq V^{T B} \equiv\left(\begin{array}{ccc}
\sqrt{2 / 3} & 1 / \sqrt{3} & 0 \\
-1 / \sqrt{6} & 1 / \sqrt{3} & 1 / \sqrt{2} \\
1 / \sqrt{6} & -1 / \sqrt{3} & +1 / \sqrt{2}
\end{array}\right)
$$

amounting to $\theta_{x}=\arcsin \left(\frac{1}{\sqrt{3}}\right), \theta_{y}=\frac{\pi}{4}$ and $\theta_{z}=0$.

The author of [6] showed that the TB pattern is equivalent to a certain form for the $M_{\nu}$ in the flavor basis called 'tripartite':

$$
M_{\nu}^{\operatorname{diag}}=\left(V^{T B}\right)^{T} M_{\nu}^{T B} V^{T B} \Leftrightarrow M_{\nu}^{T B}=M_{A}+M_{B}+M_{C},
$$


where

$$
M_{A}=A\left(\begin{array}{lll}
1 & 0 & 0 \\
0 & 1 & 0 \\
0 & 0 & 1
\end{array}\right), \quad M_{B}=B\left(\begin{array}{ccc}
-1 & 0 & 0 \\
0 & 0 & 1 \\
0 & 1 & 0
\end{array}\right), \quad M_{C}=C\left(\begin{array}{ccc}
1 & 1 & -1 \\
1 & 1 & -1 \\
-1 & -1 & 1
\end{array}\right)
$$

with neutrino eigen masses related to the tripartite coefficients via:

$$
\begin{gathered}
m_{1}=A-B, m_{2}=A-B+3 C, m_{3}=A+B, \\
A=\left(m_{1}+m_{3}\right) / 2, B=\left(m_{3}-m_{1}\right) / 2, C=\left(m_{2}-m_{1}\right) / 3 .
\end{gathered}
$$

We showed in $[7,8]$ that $M_{A}+M_{B}$ has a $U(1)$ underlying symmetry which is broken by $M_{C}$, however a residual $\left(Z_{2}\right)^{3}$ symmetry is left unbroken and this remaining symmetry (called henceforth S-symmetry) characterizes uniquely the tripartite form, and hence the TB pattern. More explicitly, we determined the set of invertible matrices $S$ which leave the neutrino mass matrix corresponding to the TB pattern unchanged under congruency (or what was called in [9] the form invariance condition):

$$
S^{T} M_{\nu} S=M_{\nu}
$$

We have found that this set includes eight elements forming a subgroup of the nonsingular $3 \times 3$ complex matrices under matrix multiplication. Moreover, this subgroup is isomorphic to the multiplicative group $\left(Z_{2}\right)^{3}$ with three generators, whence the name.

From a mathematical viewpoint, the form of $V^{T B}$ is just a unitary matrix whose elements are expressed in terms of simple numbers via algebraic operations. One would wonder thus what would be the consequences on the flavor S-symmetry had the experimental data led to different values. In [10], relations between residual $Z_{2}$ symmetries and patterns not conforming to the TB mixing pattern were studied.

Actually, and despite the phenomenological success of the TB pattern [11], it is important, in light of the recent neutrino data best fit $[2,12,13,14,15]$ amounting to $\theta_{x}=33.58^{\circ}, \theta_{y}=40.40^{\circ}$ and $\theta_{z}=8.33^{\circ}$, to restudy the question of the $\left(Z_{2}\right)^{3}$ S-symmetry, possessed by the neutrino mass matrix, in the case of TB mixing but with non-zero $\theta_{z}$ (which we would call a non-tri-bimaximal mixing pattern and denote it by 'NTB'), and find an explicit form of this flavor S-symmetry different from the specific form we found in [7] corresponding to the TB case. The object of this paper is to present such a study with the cosmological consequences for the baryogenesis.

For this, we follow the method detailed in [7] which amounts to finding the generators of the $\left(Z_{2}\right)^{3}$ S-symmetry in the diagonalized basis by imposing the form invariance condition, then deducing the corresponding generators of the $\left(Z_{2}\right)^{3}$ S-symmetry in the flavor basis. We find also a 'variant' tripartite form characterizing the NTB pattern for generic $\theta_{z}$. Although all the formulae are derived with a general $\theta_{z} \neq 0$, we nonetheless, for numerical applications and definite discussions, would specify the NTB pattern to the case $\theta_{z}=\arcsin \left(\frac{1}{\sqrt{50}}\right)$ matching, to a good approximation, the recent best fitted data, and would denote this special case by a subscript zero mark.

All the same, we stress that the special value $\theta_{z}=\arcsin \left(\frac{1}{\sqrt{50}}\right)$ is not a result from any theoretical consideration, but suggested only by experiment, whereas the general results are independent of the explicit value of $\theta_{z}$. In fact, we could as well leave the values of $\theta_{x}$ and $\theta_{y}$ as free parameters. However, the resulting expressions are cumbersome enough not to be of practical use, and we have opted to keep these two mixing angles fixed to their $\mathrm{TB}$ values consistent with experiment. This was motivated in particular by the fact that, compared to the previous oscillation data in [16], the new data (e.g. [2]) did not signal a palpable change in the values of $\theta_{x}$ and $\theta_{y}$ in contrast to $\theta_{z}$ where the former data were consistent with the value $\theta_{z}=0$ rejected now by the present data (see Table 1 ).

As in [7], we show that imposing the flavor S-symmetry in a complete lepton setting, coupled to scalar fields singlet with respect to the standard model (SM) group but with suitably chosen Yukawa couplings, can lead in a natural way to the charged lepton mass hierarchy. Moreover, by assuming the canonical type-I seesaw mechanism formula:

$$
M_{\nu}=M_{\nu}^{D} M_{R}^{-1}\left(M_{\nu}^{D}\right)^{T},
$$




\begin{tabular}{|c|c|c|c|c|c|c|c|}
\hline Parameter & Present Best fit & $\overline{\text { Present } 2 \sigma \text { range }}$ & NTB & $\mathrm{NTB}_{0}$ & Past Best fit & Past $2 \sigma$ range & $\overline{\mathrm{TB}}$ \\
\hline$\theta_{x}$ & $33.58^{\circ}$ & {$\left[31.95^{\circ}, 36.09^{\circ}\right]$} & $35.27^{\circ}$ & $35.27^{\circ}$ & $34.08^{\circ}$ & {$\left[31.11^{\circ}, 37.50^{\circ}\right]$} & $35.27^{\circ}$ \\
\hline$\theta_{y}$ & $40.40^{\circ}$ & {$\left[36.87^{\circ}, 50.77^{\circ}\right]$} & $45^{\circ}$ & $45^{\circ}$ & $41.55^{\circ}$ & {$\left[35.86^{\circ}, 51.97^{\circ}\right]$} & $45^{\circ}$ \\
\hline$\theta_{z}$ & $8.33^{\circ}$ & {$\left[6.29^{\circ}, 11.68^{\circ}\right]$} & $\theta_{z}$ & $8.13^{\circ}$ & $5.44^{\circ}$ & {$\left[0^{\circ}, 10.30^{\circ}\right]$} & $0^{\circ}$ \\
\hline
\end{tabular}

Table 1: The present ([2]) and past ([16]) global-fit results for the three neutrino mixing angles $\left(\theta_{x}, \theta_{y}, \theta_{z}\right)$, with their $2 \sigma$ ranges $(95 \%$ C.L.). We show also the corresponding values in the generic and the special NTB patterns, and in the TB pattern.

where $M_{R}$ is the heavy Majorana mass matrix for the RH neutrinos, and $M_{\nu}^{D}$ is the Dirac neutrino mass matrix, one can accommodate the different possible neutrino mass patterns provided the neutrino components transform in a specific way under the S-symmetry. However, and as was the case of the TB pattern in [7], we show that we need to call for type-II seesaw mechanism in order to account for the observed baryon to photon density.

The plan of the paper is as follows. In section 2 we find an explicit realization of the $\left(Z_{2}\right)^{3}$ S-symmetry leading to the observed NTB pattern. In section 3 we impose the symmetry on the charged leptons and discuss their mass hierarchy. In section 4 we show within type-I seesaw scenario how we can obtain the different types of neutrino mass hierarchies. We show also why we shall need in section 5 the other type-II seesaw mechanism in order to explain the baryon asymmetry generated by lepton asymmetry. We end up by a summary in section 6 .

\section{The underlying symmetry of the NTB pattern}

We review here the approach of form invariance, which we used in [7], in order to find an explicit form of the $\left(Z_{2}\right)^{3}$ S-symmetry leading to the NTB pattern defined by:

$$
\theta_{y}=\arcsin \frac{1}{\sqrt{2}}, \theta_{x}=\arcsin \frac{1}{\sqrt{3}}, \theta_{z} \neq 0,
$$

which would correspond to a flavor mixing matrix:

$$
V^{N T B}=\left(\begin{array}{ccc}
\sqrt{\frac{2}{3}} c_{z} & \frac{c_{z}}{\sqrt{3}} & s_{z} \\
-\frac{1}{\sqrt{6}}\left(1+\sqrt{2} s_{z}\right) & \frac{1}{\sqrt{3}}\left(1-\frac{s_{z}}{\sqrt{2}}\right) & \frac{c_{z}}{\sqrt{2}} \\
\frac{1}{\sqrt{6}}\left(1-\sqrt{2} s_{z}\right) & -\frac{1}{\sqrt{3}}\left(1+\frac{s_{z}}{\sqrt{2}}\right) & \frac{c_{z}}{\sqrt{2}}
\end{array}\right)
$$

We see that seeking unitary matrices $S$ satisfying the form invariance formula (Eq. 12) is equivalent to finding unitary matrices $U$ satisfying this invariance in the diagonalized basis:

$$
U^{T} M_{\nu}^{\text {diag }} U=M_{\nu}^{\text {diag }},
$$

provided

$$
S=V^{*} \cdot U \cdot V^{T}
$$

The different values of the $m_{i}$ 's lead, via Eq. (16), automatically to

$$
U=\operatorname{diag}( \pm 1, \pm 1, \pm 1)
$$

and thus, denoting by $I_{i}$ the reflection across the $i^{\text {th }}$ axis $\left(x=1, y=2, z=3\right.$ : e.g. $I_{1} \equiv I_{x}=$ $\operatorname{diag}(-1,+1,+1))$, we find the symmetries $S_{i}$ 's which characterize the NTB pattern for generic $\theta_{z}$,

$$
S_{1}=\left(V^{N T B}\right)^{*} \cdot \operatorname{diag}(-1,1,1) \cdot\left(V^{N T B}\right)^{T}=\left(\begin{array}{ccc}
1-4 c_{z}^{2} / 3 & 2 c_{z}\left(1+\sqrt{2} s_{z}\right) / 3 & 2 c_{z}\left(-1+\sqrt{2} s_{z}\right) / 3 \\
2 c_{z}\left(1+\sqrt{2} s_{z}\right) / 3 & 2\left(-\sqrt{2} s_{z}+c_{z}^{2}\right) / 3 & c_{2 z} / 3 \\
2 c_{z}\left(-1+\sqrt{2} s_{z}\right) / 3 & c_{2 z} / 3 & 2\left(\sqrt{2} s_{z}+c_{z}^{2}\right) / 3
\end{array}\right),
$$




$$
\begin{aligned}
S_{2}=\left(V^{N T B}\right)^{*} \cdot \operatorname{diag}(1,-1,1) \cdot\left(V^{N T B}\right)^{T}= & \left(\begin{array}{ccc}
1-2 c_{z}^{2} / 3 & \sqrt{2} c_{z}\left(-\sqrt{2}+s_{z}\right) / 3 & \sqrt{2} c_{z}\left(\sqrt{2}+s_{z}\right) / 3 \\
\sqrt{2} c_{z}\left(-\sqrt{2}+s_{z}\right) / 3 & \left(2 \sqrt{2} s_{z}+c_{z}^{2}\right) / 3 & \left(1+c_{z}^{2}\right) / 3 \\
\sqrt{2} c_{z}\left(\sqrt{2}+s_{z}\right) / 3 & \left(1+c_{z}^{2}\right) / 3 & \left(-2 \sqrt{2} s_{z}+c_{z}^{2}\right) / 3
\end{array}\right) \\
S_{3}=\left(V^{N T B}\right)^{*} \cdot \operatorname{diag}(1,1,-1) \cdot\left(V^{N T B}\right)^{T}= & \left(\begin{array}{ccc}
c_{2 z} & -s_{2 z} / \sqrt{2} & -s_{2 z} / \sqrt{2} \\
-s_{2 z} / \sqrt{2} & s_{z}^{2} & -c_{z}^{2} \\
-s_{2 z} / \sqrt{2} & -c_{z}^{2} & s_{z}^{2}
\end{array}\right) .
\end{aligned}
$$

The three matrices $\left(S_{i}, i=1,2,3\right)$ represent the generators of the $\left(Z_{2}\right)^{3}$-symmetry satisfied by the neutrino mass matrix.

Taking into consideration that the form invariance formula involves the matrix $S$ quadratically, and that, say, $S_{1} S_{2}=-S_{3}$, we have thus proved, for each generic value $\theta_{z}$, the following:

$$
\begin{aligned}
\left(\forall S \in\left\langle S_{1}, S_{2}, S_{3}\right\rangle, S^{T} \cdot M_{\nu} \cdot S=M_{\nu}\right) & \Leftrightarrow\left(\forall S \in\left\langle S_{1}, S_{2}\right\rangle, S^{T} \cdot M_{\nu} \cdot S=M_{\nu}\right) \\
& \Leftrightarrow\left[\left(V^{N T B}\right)^{T} \cdot M_{\nu} \cdot V^{N T B}=M_{\nu}^{\text {diag }}\right] .
\end{aligned}
$$

We would like now to find a variant of the tripartite form which would characterize the generic NTB pattern so that to generalize the equivalence formula of Eqs. $(9,10)$. For this we note that the matrix $R_{z}(\delta)$ (Eq. 5), when the phases are dropped, becomes $R_{13}\left(\theta_{z}\right)$ the rotation around the y-axis, so we have

$$
V^{N T B}=R_{23}\left(\theta_{y}\right) \cdot R_{13}\left(\theta_{z}\right) \cdot R_{12}\left(\theta_{x}\right) \quad, \quad V^{T B}=V^{N T B}\left(\theta_{z}=0\right),
$$

where the rotation matrices are given by

$$
R_{12}\left(\theta_{x}\right) \equiv\left(\begin{array}{ccc}
c_{x} & s_{x} & 0 \\
-s_{x} & c_{x} & 0 \\
0 & 0 & 1
\end{array}\right), \quad R_{23}\left(\theta_{y}\right) \equiv\left(\begin{array}{ccc}
1 & 0 & 0 \\
0 & c_{y} & s_{y} \\
0 & -s_{y} & c_{y}
\end{array}\right) \quad R_{13}\left(\theta_{z}\right) \equiv\left(\begin{array}{ccc}
c_{z} & 0 & s_{z} \\
0 & 1 & 0 \\
-s_{z} & 0 & c_{z}
\end{array}\right)
$$

We get then

$$
\begin{aligned}
V^{N T B} & =W \cdot V^{T B} \\
W & =R_{23}\left(\theta_{y}=\frac{\pi}{4}\right) R_{13}\left(\theta_{z}\right) R_{23}^{-1}\left(\theta_{y}=\frac{\pi}{4}\right)=\left(\begin{array}{ccc}
c_{z} & \frac{s_{z}}{\sqrt{2}} & \frac{s_{z}}{\sqrt{2}} \\
-\frac{s_{z}}{\sqrt{2}} & \frac{1+c_{z}}{2} & -\frac{1-c_{z}}{2} \\
-\frac{s_{z}}{\sqrt{2}} & -\frac{1-c_{z}}{2} & \frac{1+c_{z}}{2}
\end{array}\right) .
\end{aligned}
$$

Using the equality,

$$
\left(V^{T B}\right)^{T} \cdot M_{\nu}^{T B} \cdot V^{T B}=\left(V^{N T B}\right)^{T} \cdot M_{\nu}^{N T B} \cdot V^{N T B}=M_{\nu}^{\operatorname{diag}} \equiv \operatorname{diag}\left(m_{1}, m_{2}, m_{3}\right),
$$

we can relate the neutrino mass matrices in the NTB and the TB patterns with each other in the following way:

$$
M_{\nu}^{N T B}=W \cdot M_{\nu}^{T B} \cdot W^{T} .
$$

At this stage, it would be useful if the sought form for $M_{\nu}^{N T B}$ characterizing uniquely the generic NTB pattern varies slightly from the tripartite form (Eqs. 9, 10), and hence called hereafter a 'generic' modified tripartite form. To fix the ideas, we choose a pattern where the diagonal elements of $M_{\nu}^{N T B}$ 'resemble' their counterparts in the tripartite form of $M_{\nu}^{T B}$, in that we impose:

$$
\begin{aligned}
& M_{\nu}^{N T B}(1,1)=A^{z}-B^{z}+C^{z} \\
& M_{\nu}^{N T B}(2,2)=A^{z}+C^{z} \\
& M_{\nu}^{N T B}(3,3)=A^{z}-C^{z}
\end{aligned}
$$

Now, by equating the diagonal elements in both sides of Eq. (25), one can express the $\left(A^{z}, B^{z}, C^{z}\right)$ in terms of the tripartite form coefficients $(A, B, C)$, then by inverting these expressions one can find the 
non-diagonal elements of $M_{\nu}^{N T B}$ in terms of $\left(A^{z}, B^{z}, C^{z}\right)$ :

$$
\begin{aligned}
& M_{\nu}^{N T B}(1,2)=\frac{\sqrt{2} s_{z} c_{z}}{1-3 s_{z}^{2}} B^{z}+\frac{c_{z}\left(1-2 \sqrt{2} s_{z}\right)}{2\left(1-3 s_{z}^{2}\right)} C^{z}-\frac{c_{z}}{\sqrt{2} s_{z}} C^{z} \\
& M_{\nu}^{N T B}(1,3)=\frac{\sqrt{2} s_{z} c_{z}}{1-3 s_{z}^{2}} B^{z}+\frac{c_{z}\left(1-2 \sqrt{2} s_{z}\right)}{2\left(1-3 s_{z}^{2}\right)} C^{z}+\frac{c_{z}}{\sqrt{2} s_{z}} C^{z} \\
& M_{\nu}^{N T B}(2,3)=\frac{c_{z}^{2}}{1-3 s_{z}^{2}} B^{z}+\frac{-2 s_{z} c_{z}^{2}+2 \sqrt{2} c_{z}^{2}-\sqrt{2}}{2 s_{z}\left(1-3 s_{z}^{2}\right)} C^{z} .
\end{aligned}
$$

The relations in Eqs. $(26,27)$ define the generic modified tripartite form for the symmetric neutrino mass matrix.

This pattern of $M_{\nu}^{N T B}$ is characterized also by some 'mass relations' relating its different entries:

$$
\begin{aligned}
M_{\nu}^{N T B}(1,2)+M_{\nu}^{N T B}(1,3)= & \frac{\tan 2 \theta_{z}}{2}\left[M_{\nu}^{N T B}(2,3)-M_{\nu}^{N T B}(1,1)+\frac{M_{\nu}^{N T B}(2,2)+M_{\nu}^{N T B}(3,3)}{2}\right], \\
M_{\nu}^{N T B}(2,2)-M_{\nu}^{N T B}(3,3)= & \sqrt{2} \tan \theta_{z}\left[M_{\nu}^{N T B}(1,3)-M_{\nu}^{N T B}(1,2)\right], \\
2 M_{\nu}^{N T B}(1,1)+6 M_{\nu}^{N T B}(2,2)= & {\left[M_{\nu}^{N T B}(3,3)+M_{\nu}^{N T B}(2,2)\right]\left(1+\cos 2 \theta_{z}\right)-2\left[M_{\nu}^{N T B}(1,1)-M_{\nu}^{N T B}(2,3)\right] \cos 2 \theta_{z} } \\
& -2\left[M_{\nu}^{N T B}(1,2)+M_{\nu}^{N T B}(1,3)\right] \cos 2 \theta_{z}-2\left[M_{\nu}^{N T B}(1,2)-M_{\nu}^{N T B}(1,3)\right] \cos \theta_{z} \\
& -2\left[M_{\nu}^{N T B}(3,3)-M_{\nu}^{N T B}(2,2)\right] \cos \theta_{z}+2 \sqrt{2}\left[M_{\nu}^{N T B}(1,2)+M_{\nu}^{N T B}(1,3)\right] \sin 2 \theta_{z} \\
& +\frac{1}{\sqrt{2}}\left[M_{\nu}^{N T B}(2,2)+M_{\nu}^{N T B}(3,3)\right] \sin 2 \theta_{z}-\sqrt{2}\left[M_{\nu}^{N T B}(1,1)-M_{\nu}^{N T B}(2,3)\right] \sin 2 \theta_{z} \\
& -2 \sqrt{2}\left[M_{\nu}^{N T B}(1,3)-M_{\nu}^{N T B}(1,2)\right] \sin \theta_{z}-\sqrt{2}\left[M_{\nu}^{N T B}(3,3)-M_{\nu}^{N T B}(2,2)\right] \sin \theta_{z} .
\end{aligned}
$$

These relations are to be contrasted with the simple ones characterizing the TB pattern $\left(\theta_{z}=0\right)$ :

$$
\begin{aligned}
M_{\nu}^{T B}(1,2)+M_{\nu}^{T B}(1,3) & =0, \\
M_{\nu}^{T B}(2,2)-M_{\nu}^{T B}(3,3) & =0, \\
M_{\nu}^{T B}(1,1)+M_{\nu}^{T B}(1,2)-M_{\nu}^{T B}(2,2)+M_{\nu}^{T B}(2,3) & =0 .
\end{aligned}
$$

Up till now, the obtained results hold independently of an explicit value of $\theta_{z}$. However, it is useful now, in order to contrast our analysis with the experimental data, to specify our results to the precise value $\left(\theta_{z} \simeq 8.13^{\circ} \simeq \arcsin \frac{1}{\sqrt{50}}\right)$. In this specific case, we have the 'observed' mixing matrix:

$$
V_{0}^{N T B}=\left(\begin{array}{ccc}
\frac{7}{15} \sqrt{3} & \frac{7}{30} \sqrt{6} & \frac{1}{10} \sqrt{2} \\
-\frac{1}{5} \sqrt{6} & \frac{3}{10} \sqrt{3} & \frac{7}{10} \\
\frac{2}{15} \sqrt{6} & -\frac{11}{30} \sqrt{3} & \frac{7}{10}
\end{array}\right)
$$

As to the $\left(Z_{2}\right)^{3}$ generators for the S-symmetry, they are given now as follows.

$$
S_{01}=\left(\begin{array}{ccc}
-\frac{23}{75} & \frac{14 \sqrt{2}}{25} & -\frac{28 \sqrt{2}}{75} \\
\frac{14 \sqrt{2}}{25} & \frac{13}{25} & \frac{8}{25} \\
-\frac{28 \sqrt{2}}{75} & \frac{8}{25} & \frac{59}{75}
\end{array}\right), S_{02}=\left(\begin{array}{ccc}
\frac{26}{75} & -\frac{21 \sqrt{2}}{50} & \frac{77 \sqrt{2}}{150} \\
-\frac{21 \sqrt{2}}{50} & \frac{23}{50} & \frac{33}{50} \\
\frac{77 \sqrt{2}}{150} & \frac{33}{50} & \frac{29}{150}
\end{array}\right), S_{03}=\left(\begin{array}{ccc}
\frac{24}{25} & -\frac{7 \sqrt{2}}{50} & -\frac{7 \sqrt{2}}{50} \\
-\frac{7 \sqrt{2}}{50} & \frac{1}{50} & -\frac{49}{50} \\
-\frac{7 \sqrt{2}}{50} & -\frac{49}{50} & \frac{1}{50}
\end{array}\right) .
$$

We also find the transition matrix between the TB and our special NTB patterns as equal to

$$
W_{0}=\left(\begin{array}{ccc}
\frac{7 \sqrt{2}}{10} & \frac{1}{10} & \frac{1}{10} \\
-\frac{1}{10} & \frac{7 \sqrt{2}+10}{20} & \frac{7 \sqrt{2}-10}{20} \\
-\frac{1}{10} & \frac{7 \sqrt{2}-10}{20} & \frac{7 \sqrt{2}+10}{20}
\end{array}\right)
$$

which corresponds to the NTB 'special' modified tripartite form

$$
M_{\nu 0}^{N T B}=\left(\begin{array}{ccc}
A_{0}-B_{0}+C_{0} & \frac{7 \sqrt{2}}{47}\left(B_{0}-22 C_{0}\right) & \frac{7 \sqrt{2}}{47}\left(B_{0}+25 C_{0}\right) \\
\frac{7 \sqrt{2}}{47}\left(B_{0}-22 C_{0}\right) & A_{0}+C_{0} & \frac{1}{47}\left(49 B_{0}+191 C_{0}\right) \\
\frac{7 \sqrt{2}}{47}\left(B_{0}+25 C_{0}\right) & \frac{1}{47}\left(49 B_{0}+191 C_{0}\right) & A_{0}-C_{0}
\end{array}\right)
$$


where the neutrino eigen masses and the coefficients of this special modified tripartite form are related by:

$$
\begin{array}{lll}
m_{1}=A_{0}-\frac{49}{47} B_{0}+\frac{279}{47} C_{0} & , & A_{0}=\frac{49}{100} m_{3}+\frac{101}{300} m_{2}+\frac{13}{75} m_{1}, \\
m_{2}=A_{0}-\frac{49}{47} B_{0}-\frac{426}{47} C_{0} & , & B_{0}=\frac{47}{100} m_{3}-\frac{17}{300} m_{2}-\frac{31}{75} m_{1}, \\
m_{3}=A_{0}+\frac{51}{47} B_{0}+\frac{194}{47} C_{0} & , & C_{0}=-\frac{1}{15} m_{2}+\frac{1}{15} m_{1} .
\end{array}
$$

The resulting mass spectra can accommodate all types of neutrino mass hierarchies as follows;

- Normal hierarchy: with

$$
A_{0} \simeq B_{0}, \quad C_{0} \ll B_{0},
$$

we get a mass spectrum corresponding to normal hierarchy:

$$
\left[m_{1}, m_{2}, m_{3}\right] \simeq\left[A_{0}-\frac{49}{47} B_{0}, A_{0}-\frac{49}{47} B_{0}, A_{0}+\frac{51}{47} B_{0}\right] .
$$

- Inverted hierarchy: with

$$
A_{0} \simeq-B_{0}, \quad C_{0} \ll B_{0},
$$

we get a mass spectrum corresponding to inverted hierarchy:

$$
\left[m_{1}, m_{2}, m_{3}\right] \simeq\left[A_{0}-\frac{49}{47} B_{0}, A_{0}-\frac{49}{47} B_{0}, \frac{194}{47} C_{0}\right] .
$$

- Degenerate case: with

$$
A_{0} \gg B_{0} \gg C_{0}
$$

we get a quasi degenerate mass spectrum

$$
\left[m_{1}, m_{2}, m_{3}\right] \simeq\left[A_{0}, A_{0}, A_{0}\right]
$$

In addition to what precedes, we could check that the symmetry generated by the generic $\left(S_{1}, S_{2}, S_{3}\right)$ (or by any two of the three elements) is equivalent to the generic modified tripartite form. In particular, and limiting our attention to our special case $\left(\theta_{z}=\arcsin \frac{1}{\sqrt{50}}\right)$ we have the following equivalences corresponding to the special modified tripartite form:

$$
\begin{aligned}
{\left[\left(S_{0 i}\right)^{T} \cdot M_{\nu 0} \cdot S_{0 i}=M_{\nu 0}\right] } & \Leftrightarrow\left\{k \neq l \Rightarrow\left[\left(V_{0}^{N T B}\right)^{T} \cdot M_{\nu 0} \cdot V_{0}^{N T B}\right]_{k l}=0\right\} \\
& \Leftrightarrow\left[\exists A_{0}, B_{0}, C_{0}: M_{\nu 0}=\left(\begin{array}{ccc}
A_{0}-B_{0}+C_{0} & \frac{7 \sqrt{2}}{47}\left(B_{0}-22 C_{0}\right) & \frac{7 \sqrt{2}}{47}\left(B_{0}+25 C_{0}\right) \\
\frac{7 \sqrt{2}}{47}\left(B_{0}-22 C_{0}\right) & A_{0}+C_{0} & \frac{1}{47}\left(49 B_{0}+191 C_{0}\right) \\
\frac{7 \sqrt{2}}{47}\left(B_{0}+25 C_{0}\right) & \frac{1}{47}\left(49 B_{0}+191 C_{0}\right) & A_{0}-C_{0}
\end{array}\right)\right]
\end{aligned}
$$

where $i$ spans the set $\{1,2,3\}$ or any two different elements in it.

\section{The charged-lepton mass matrix}

Up to this point, we have found explicit realizations of the $\left(Z_{2}\right)^{3}$ S-symmetry which would lead to the phenomenologically interesting form for $M_{\nu}$ corresponding to the NTB pattern. However, we would 
now lift our findings to an underlying symmetry level and construct a model for the leptons where the Lagrangian is kept invariant under the flavor S-symmetry.

We see that the SM term

$$
\mathcal{L}_{1}=Y_{i j} \bar{L}_{i} \Phi l_{j}^{c}
$$

has to be absent provided the SM-singlet charged RH leptons $l_{j}^{c}$ and the SM Higgs are singlet under $S=\left(Z_{2}\right)^{3}$, whereas the left doublets transform componentwise as:

$$
L_{i} \rightarrow S_{i j} L_{j}
$$

To show this, it is sufficient to note that the invariance of $\mathcal{L}_{1}$ under $S$ implies the matrix equation

$$
S^{T} \cdot Y=Y,
$$

which can be only satisfied for a vanishing $Y$. Had we restricted the symmetry to just two factors, for example $\left\langle S_{1}, S_{2}\right\rangle$, this term would have been allowed. However, in this case corresponding to the flavor S-symmetry being an $\left(Z_{2}\right)^{2}$-symmetry, one can check that the charged lepton squared mass matrix, proportional to $Y \cdot Y^{\dagger}$ when the SM Higgs gets its vacuum expectation value (vev), would be singular with two zero eigen values, and the charged lepton mass hierarchy can not thus be produced. Another serious drawback for the existence of such a term in $\left(Z_{2}\right)^{2}$-symmetry is that the left handed charged leptons need here to be rotated non-trivially in order to achieve the diagonalization of the charged lepton mass matrix, and this in turn would destroy the prediction of the NTB pattern in Eq. (30).

In order to remedy this, we add three SM-singlet scalar fields $\Delta_{k}$ coupled to the lepton LH doublets through the dimension 5 operator:

$$
\mathcal{L}_{2}=\frac{f_{i k r}}{\Lambda} \bar{L}_{i} \Phi \Delta_{k} l_{r}^{c}
$$

and we assume $\Delta_{k}$ to vary under S-symmetry as

$$
\Delta_{i} \rightarrow S_{i j} \Delta_{j}
$$

As in [7], this ad hoc assumption of $\mathcal{L}_{2}$ with only one Higgs field is suitable to reduce the effects of flavor changing neutral currents [17]. Invariance of $\mathcal{L}_{2}$ under S-symmetry leads to:

$$
S^{T} f_{r} S=f_{r}
$$

where $\left(f_{r}\right)_{i j}=f_{i j r}$. Thus, from Eq. 41 , the matrix $f_{r}$ has the NTB modified tripartite form:

$$
f_{r}=\left(\begin{array}{ccc}
A_{0}^{r}-B_{0}^{r}+C_{0}^{r} & \frac{7 \sqrt{2}}{47}\left(B_{0}^{r}-22 C_{0}^{r}\right) & \frac{7 \sqrt{2}}{47}\left(B_{0}^{r}+25 C_{0}^{r}\right) \\
\frac{7 \sqrt{2}}{47}\left(B_{0}^{r}-22 C_{0}^{r}\right) & A_{0}^{r}+C_{0}^{r} & \frac{1}{47}\left(49 B_{0}^{r}+191 C_{0}^{r}\right) \\
\frac{7 \sqrt{2}}{47}\left(B_{0}^{r}+25 C_{0}^{r}\right) & \frac{1}{47}\left(49 B_{0}^{r}+191 C_{0}^{r}\right) & A_{0}^{r}-C_{0}^{r}
\end{array}\right) .
$$

When $\Delta_{k}$ and $\phi^{\circ}$ take the vevs $\left\langle\Delta_{k}\right\rangle=\delta_{k}, v=\left\langle\phi^{\circ}>\right.$, then $\mathcal{L}_{2}$ would generate charged lepton mass matrix:

$$
\left(\mathcal{M}_{l}\right)_{i r}=\frac{v f_{i k r}}{\Lambda} \delta_{k}
$$

One can arrange the vevs and the Yukawa couplings such that $\mathcal{M}_{l}$, after suitably rotating the flavor- and SM-singlets $l_{j}^{c}$, is the charged lepton mass matrix in the flavor basis. For example, if $\delta_{1}, \delta_{2} \ll \delta_{3}$ we get

$$
M_{l}=\frac{v \delta_{3}}{\Lambda}\left(\begin{array}{lll}
A_{1}^{\prime} & A_{2}^{\prime} & A_{3}^{\prime} \\
B_{1}^{\prime} & B_{2}^{\prime} & B_{3}^{\prime} \\
C_{1}^{\prime} & C_{2}^{\prime} & C_{3}^{\prime}
\end{array}\right)
$$

where

$$
A_{i}^{\prime}=\frac{7 \sqrt{2}}{47}\left(B_{0}^{i}+25 C_{0}^{i}\right) \quad, \quad B_{i}^{\prime}=\frac{1}{47}\left(49 B_{0}^{i}+191 C_{0}^{i}\right) \quad, \quad C_{i}^{\prime}=A_{0}^{i}-C_{0}^{i}
$$


The charged lepton squared mass matrix $\left(M_{l} M_{l}^{\dagger}\right)$ assumes the form,

$$
M_{l} M_{l}^{\dagger} \approx\left(\frac{v \delta_{3}}{\Lambda}\right)^{2}\left(\begin{array}{lll}
\mathbf{A}^{\prime} \cdot \mathbf{A}^{\prime} & \mathbf{A}^{\prime} \cdot \mathbf{B}^{\prime} & \mathbf{A}^{\prime} \cdot \mathbf{C}^{\prime} \\
\mathbf{B}^{\prime} \cdot \mathbf{A}^{\prime} & \mathbf{B}^{\prime} \cdot \mathbf{B}^{\prime} & \mathbf{B}^{\prime} \cdot \mathbf{C}^{\prime} \\
\mathbf{C}^{\prime} \cdot \mathbf{A}^{\prime} & \mathbf{C}^{\prime} \cdot \mathbf{B}^{\prime} & \mathbf{C}^{\prime} \cdot \mathbf{C}^{\prime}
\end{array}\right)
$$

where $\mathbf{A}^{\prime}$ is the complex vector of components $A_{i}^{\prime}\left(i=1,2,3\right.$, similarly for $\left.\mathbf{B}^{\prime}, \mathbf{C}^{\prime}\right)$ and the usual inner product of two complex vectors $\left(\mathbf{A}^{\prime}\right.$ and $\left.\mathbf{B}^{\prime}\right)$ is defined as $\mathbf{A}^{\prime} \cdot \mathbf{B}^{\prime} \equiv \sum_{i=1}^{3} A_{i}^{\prime}{ }_{i} B_{i}^{\prime *}$. The charged lepton mass matrix, $M_{l}$, is nonsingular provided the three vectors $\left(\mathbf{A}^{\prime}, \mathbf{B}^{\prime}, \mathbf{C}^{\prime}\right)$ are linearly independent (amounting to non-coplanar vectors in the real Euclidian case).

In order to show that $M_{l}$ can naturally represent the lepton mass matrix in the flavor basis, let us just assume the magnitudes of the three vectors coming in ratios comparable to the lepton mass ratios:

$$
\frac{\left|\mathbf{A}^{\prime}\right|}{\left|\mathbf{C}^{\prime}\right|} \equiv \lambda_{e} \sim \frac{m_{e}}{m_{\tau}}=2.8 \times 10^{-4} \quad, \quad \frac{\left|\mathbf{B}^{\prime}\right|}{\left|\mathbf{C}^{\prime}\right|} \equiv \lambda_{\mu} \sim \frac{m_{\mu}}{m_{\tau}}=5.9 \times 10^{-2},
$$

where $\left|\mathbf{A}^{\prime}\right|$ represent the norm of the vector defined by,

$$
\left|\mathbf{A}^{\prime}\right|=\sqrt{\mathbf{A}^{\prime} \cdot \mathbf{A}^{\prime}}, \quad\left(\text { similarly for } \mathbf{B}^{\prime}, \mathbf{C}^{\prime}\right) .
$$

This leads the squared mass matrix to be written as:

$$
Q_{\lambda} \equiv M_{l} M_{l}^{\dagger} \approx\left(\frac{v \delta_{3}}{\Lambda}\right)^{2}\left|\mathbf{C}^{\prime}\right|^{2}\left(\begin{array}{ccc}
\lambda_{e}^{2} & \lambda_{e} \lambda_{\mu} \cos \psi e^{i \alpha} & \lambda_{e} \cos \phi e^{i \beta} \\
\lambda_{e} \lambda_{\mu} \cos \psi e^{-i \alpha} & \lambda_{\mu}^{2} & \lambda_{\mu} \cos \theta e^{i \gamma} \\
\lambda_{e} \cos \phi e^{-i \beta} & \lambda_{\mu} \cos \theta e^{-i \gamma} & 1
\end{array}\right)
$$

where $\psi, \phi$ and $\theta$ are the "angles" between the pairs of complex vectors $\left(\mathbf{A}^{\prime}, \mathbf{B}^{\prime}\right),\left(\mathbf{A}^{\prime}, \mathbf{C}^{\prime}\right)$ and $\left(\mathbf{C}^{\prime}, \mathbf{B}^{\prime}\right)$ respectively, whereas $\alpha, \beta$ and $\gamma$ are the phases of the corresponding inner products ${ }^{1}$. The diagonalization of $M_{l} M_{l}^{\dagger}$ by means of an infinitesimal "rotation" amounts to seeking an antihermitian matrix

$$
I_{\epsilon}=\left(\begin{array}{ccc}
0 & \epsilon_{1} & \epsilon_{2} \\
-\epsilon_{1}^{*} & 0 & \epsilon_{3} \\
-\epsilon_{2}^{*} & -\epsilon_{3}^{*} & 0
\end{array}\right)
$$

with small parameters $\epsilon^{\prime} \mathrm{s}$, satisfying:

$$
\left(Q_{\lambda}+\left[Q_{\lambda}, I_{\epsilon}\right]\right)_{i j}=0, \quad i \neq j .
$$

If we solve this equation analytically to express the $\epsilon$ 's in terms of $\left(\lambda_{e, \mu}, \cos (\psi, \phi, \theta), \alpha, \beta\right.$, $\left.\gamma\right)$, we find, apart from "fine tuned" situations corresponding to coplanar vectors $\mathbf{A}^{\prime}, \mathbf{B}^{\prime}, \mathbf{C}^{\prime}$, that we get: $\left|\epsilon_{3}\right| \sim$ $\lambda_{\mu},\left|\epsilon_{2}\right| \sim \lambda_{e}$ and $\left|\epsilon_{1}\right| \sim \lambda_{e} / \lambda_{\mu}$, which points to a consistent solution diagonalizing $Q_{\lambda}$ close to the identity matrix given by $U_{L}^{l}=e^{I_{\epsilon}} \approx I+I_{\epsilon}$. The eigenvalues for $M_{l} M_{l}^{\dagger}$ can be approximated up to leading order in $\lambda$ 's, and by identifying them with the observed charged lepton squared masses we get,

$$
\begin{aligned}
m_{e}^{2}= & \frac{\mu^{2} \lambda_{e}^{2}}{\sin ^{2} \theta}\left(1-2 \cos ^{2} \psi+6 \cos (\delta-\beta-\alpha) \cos \psi \cos \theta \cos \phi-\cos ^{2} \theta-2 \cos ^{2} \phi \cos ^{2} \theta-2 \cos ^{2} \phi\right), \\
m_{\mu}^{2}= & \frac{\mu^{2} \lambda_{\mu}^{2}}{\sin ^{2} \theta}\left(1-3 \cos ^{2} \theta+2 \cos ^{4} \theta\right) \\
& -\frac{\mu^{2} \lambda_{e}^{2}}{\sin ^{2} \theta}\left(1-2 \cos ^{2} \psi+6 \cos (\delta-\beta-\alpha) \cos \psi \cos \theta \cos \phi-\cos ^{2} \phi-2 \cos ^{2} \phi \cos ^{2} \theta-2 \cos ^{2} \theta\right), \\
m_{\tau}^{2}= & \mu^{2}\left(1+2 \lambda_{\mu}^{2} \cos ^{2} \theta+2 \lambda_{e}^{2} \cos ^{2} \phi\right),
\end{aligned}
$$

where $\mu=\left(\frac{v \delta_{3}}{\Lambda}\right)\left|\mathbf{C}^{\prime}\right|$. By giving some fixed values to the angles and phases, one can in general solve the above mentioned equations for $\left(\lambda_{\mu}, \lambda_{e}\right.$, and $\left.\mu\right)$. For illustrative purpose, we choose a common value

\footnotetext{
${ }^{1}$ The "angle" $\psi$ and "phase" $\alpha$ between two complex vectors $\mathbf{A}^{\prime}$ and $\mathbf{B}^{\prime}$ are defined, following Cauchy-Schwartz inequality, as $\left|\mathbf{A}^{\prime} \cdot \mathbf{B}^{\prime}\right|=\left|\mathbf{A}^{\prime}\right|\left|\mathbf{B}^{\prime}\right| \cos \psi$, so we have $\mathbf{A}^{\prime} \cdot \mathbf{B}^{\prime}=\left|\mathbf{A}^{\prime}\right|\left|\mathbf{B}^{\prime}\right| \cos \psi e^{i \alpha}$, where $\alpha=\arg \left(\mathbf{A}^{\prime} \cdot \mathbf{B}^{\prime}\right)$.
} 
$\pi / 3$ for angles but for phases we have representative values as $\left(\alpha=\frac{\pi}{3}, \beta=\frac{\pi}{4}\right.$, and $\left.\gamma=\frac{\pi}{5}\right)$. The resulting solutions for $\left(\lambda_{\mu}, \lambda_{e}\right.$, and $\left.\mu\right)$ are,

$$
\lambda_{e}=7.99 \times 10^{-4}, \quad \lambda_{\mu}=8.4 \times 10^{-2}, \quad \mu=1776.978 \mathrm{MeV},
$$

while the 'exact' unitary diagonalizing matrix is given by:

$$
U_{L}^{l} \sim\left(\begin{array}{ccc}
1 & 5.46 \times 10^{-3} \exp (0.693 i \pi) & 5.41 \times 10^{-4} \exp (0.126 i \pi) \\
-5.46 \times 10^{-3} \exp (-0.693 i \pi) & 1 & 4.23 \times 10^{-2} \exp (0.20 i \pi) \\
-5.41 \times 10^{-4} \exp (-0.126 i \pi) & -4.23 \times 10^{-2} \exp (-0.20 i \pi) & 1
\end{array}\right)
$$

The deviations due to the rotations are generally small, and thus produce tiny but acceptable modifications to the mixing and phase angles of the unitary mixing matrix in Eq. (30).

\section{The NTB neutrino mass matrix and type-I seesaw scenario}

Here, the type-I seesaw formula (Eq. 13) applies. The Dirac neutrino mass matrix comes from

$$
g_{i j} \bar{L}_{i} \tilde{\Phi} \nu_{R j}
$$

where $\tilde{\Phi}=i \tau_{2} \Phi^{*}$, and we assume the RH neutrinos transforming under $S$ as

$$
\nu_{R j} \rightarrow S_{j \gamma} \nu_{R \gamma}
$$

Then, the invariance of the Lagrangian under S-symmetry leads to

$$
S^{T} \cdot g \cdot S=g .
$$

Thus we have the NTB modified tripartite form for $g$, and when $\tilde{\Phi}$ takes a vev we obtain the Dirac mass matrix as:

$$
M_{\nu 0}^{D}=v\left(\begin{array}{ccc}
A_{0}^{D}-B_{0}^{D}+C_{0}^{D} & \frac{7 \sqrt{2}}{47}\left(B_{0}^{D}-22 C_{0}^{D}\right) & \frac{7 \sqrt{2}}{47}\left(B_{0}^{D}+25 C_{0}^{D}\right) \\
\frac{7 \sqrt{2}}{47}\left(B_{0}^{D}-22 C_{0}^{D}\right) & A_{0}^{D}+C_{0}^{D} & \frac{1}{47}\left(49 B_{0}^{D}+191 C_{0}^{D}\right) \\
\frac{7 \sqrt{2}}{47}\left(B_{0}^{D}+25 C_{0}^{D}\right) & \frac{1}{47}\left(49 B_{0}^{D}+191 C_{0}^{D}\right) & A_{0}^{D}-C_{0}^{D}
\end{array}\right) .
$$

Again, the invariance under S-symmetry of the term $\frac{1}{2} \nu_{i R}^{T} C\left(M_{R}\right)_{i j} \nu_{j R}(C$ is the charge conjugation matrix) would impose the NTB modified tripartite form for the Majorana RH neutrino mass matrix:

$$
M_{R 0}=\Lambda_{R}\left(\begin{array}{ccc}
A_{0}^{R}-B_{0}^{R}+C_{0}^{R} & \frac{7 \sqrt{2}}{47}\left(B_{0}^{R}-22 C_{0}^{R}\right) & \frac{7 \sqrt{2}}{47}\left(B_{0}^{R}+25 C_{0}^{R}\right) \\
\frac{7 \sqrt{2}}{47}\left(B_{0}^{R}-22 C_{0}^{R}\right) & A_{0}^{R}+C_{0}^{R} & \frac{1}{47}\left(49 B_{0}^{R}+191 C_{0}^{R}\right) \\
\frac{7 \sqrt{2}}{47}\left(B_{0}^{R}+25 C_{0}^{R}\right) & \frac{1}{47}\left(49 B_{0}^{R}+191 C_{0}^{R}\right) & A_{0}^{R}-C_{0}^{R}
\end{array}\right) .
$$

where $\Lambda_{R}$ is a high scale characterizing the seesaw mechanism.

Thus we get also an NTB modified tripartite form for the effective neutrino mass matrix:

$$
M_{\nu 0}=\frac{v^{2}}{\Lambda_{R}}\left(\begin{array}{ccc}
A_{\nu 0}-B_{\nu 0}+C_{\nu 0} & \frac{7 \sqrt{2}}{47}\left(B_{\nu 0}-22 C_{\nu 0}\right) & \frac{7 \sqrt{2}}{47}\left(B_{\nu 0}+25 C_{\nu 0}\right) \\
\frac{7 \sqrt{2}}{47}\left(B_{\nu 0}-22 C_{\nu 0}\right) & A_{\nu 0}+C_{\nu 0} & \frac{1}{47}\left(49 B_{\nu 0}+191 C_{\nu 0}\right) \\
\frac{7 \sqrt{2}}{47}\left(B_{\nu 0}+25 C_{\nu 0}\right) & \frac{1}{47}\left(49 B_{\nu 0}+191 C_{\nu 0}\right) & A_{\nu 0}-C_{\nu 0}
\end{array}\right),
$$

where $A_{\nu 0}, B_{\nu 0}$ and $C_{\nu 0}$ are given by,

$$
\begin{aligned}
& A_{\nu 0}=\frac{13}{75} \frac{\left(A_{0}^{D}-\frac{49}{47} B_{0}^{D}+\frac{279}{47} C_{0}^{D}\right)^{2}}{A_{0}^{R}-\frac{49}{47} B_{0}^{R}+\frac{279}{47} C_{0}^{R}}+\frac{101}{300} \frac{\left(A_{0}^{D}-\frac{49}{47} B_{0}^{D}-\frac{426}{47} C_{0}^{D}\right)^{2}}{A_{0}^{R}-\frac{49}{47} B_{0}^{R}-\frac{426}{47} C_{0}^{R}}+\frac{49}{100} \frac{\left(A_{0}^{D}+\frac{51}{47} B_{0}^{D}+\frac{194}{47} C_{0}^{D}\right)^{2}}{A_{0}^{R}+\frac{51}{47} B_{0}^{R}+\frac{194}{47} C_{0}^{R}}, \\
& B_{\nu 0}=-\frac{31}{75} \frac{\left(A_{0}^{D}-\frac{49}{47} B_{0}^{D}+\frac{279}{47} C_{0}^{D}\right)^{2}}{A_{0}^{R}-\frac{49}{47} B_{0}^{R}+\frac{279}{47} C_{0}^{R}}-\frac{17}{300} \frac{\left(A_{0}^{D}-\frac{49}{47} B_{0}^{D}-\frac{426}{47} C_{0}^{D}\right)^{2}}{A_{0}^{R}-\frac{49}{47} B_{0}^{R}-\frac{426}{47} C_{0}^{R}}+\frac{47}{100} \frac{\left(A_{0}^{D}+\frac{51}{47} B_{0}^{D}+\frac{194}{47} C_{0}^{D}\right)^{2}}{A_{0}^{R}+\frac{51}{47} B_{0}^{R}+\frac{194}{47} C_{0}^{R}}, \\
& C_{\nu 0}=\frac{1}{15} \frac{\left(A_{0}^{D}-\frac{49}{47} B_{0}^{D}+\frac{279}{47} C_{0}^{D}\right)^{2}}{A_{0}^{R}-\frac{49}{47} B_{0}^{R}+\frac{279}{47} C_{0}^{R}}-\frac{1}{15} \frac{\left(A_{0}^{D}-\frac{49}{47} B_{0}^{D}-\frac{426}{47} C_{0}^{D}\right)^{2}}{A_{0}^{R}-\frac{49}{47} B_{0}^{R}-\frac{426}{47} C_{0}^{R}} .
\end{aligned}
$$


The eigenvalues of the light neutrino mass matrix in Eq. (66) are found to be:

$m_{1}=\frac{v^{2}}{\Lambda_{R}} \frac{\left(A_{0}^{D}-\frac{49}{47} B_{0}^{D}+\frac{279}{47} C_{0}^{D}\right)^{2}}{A_{0}^{R}-\frac{49}{47} B_{0}^{R}+\frac{279}{47} C_{0}^{R}}, \quad m_{2}=\frac{v^{2}}{\Lambda_{R}} \frac{\left(A_{0}^{D}-\frac{49}{47} B_{0}^{D}-\frac{426}{47} C_{0}^{D}\right)^{2}}{A_{0}^{R}-\frac{49}{47} B_{0}^{R}-\frac{426}{47} C_{0}^{R}}, \quad m_{3}=\frac{v^{2}}{\Lambda_{R}} \frac{\left(A_{0}^{D}+\frac{51}{47} B_{0}^{D}+\frac{194}{47} C_{0}^{D}\right)^{2}}{A_{0}^{R}+\frac{51}{47} B_{0}^{R}+\frac{194}{47} C_{0}^{R}}$.

As mentioned before (see Eq. 34), but now specifying to the seesaw model, all types of neutrino mass hierarchies can be accommodated as follows.

- Normal hierarchy: with

$$
A_{0}^{i} \simeq B_{0}^{i}, C_{0}^{i} \ll B_{0}^{i}, i=R, D
$$

we get a mass spectrum corresponding to normal hierarchy:

$$
\left[m_{1}, m_{2}, m_{3}\right] \simeq \frac{v^{2}}{\Lambda_{R}}\left[\frac{\left(C_{0}^{D}\right)^{2}}{C_{0}^{R}}, \frac{\left(C_{0}^{D}\right)^{2}}{C_{0}^{R}}, \frac{\left(A_{0}^{D}\right)^{2}}{A_{0}^{R}}\right]
$$

- Inverted hierarchy: with

$$
A_{0}^{i} \simeq-B_{0}^{i}, C_{0}^{i} \ll B_{0}^{i}, i=R, D
$$

we get a mass spectrum corresponding to inverted hierarchy:

$$
\left[m_{1}, m_{2}, m_{3}\right] \simeq \frac{v^{2}}{\Lambda_{R}}\left[\frac{\left(A_{0}^{D}\right)^{2}}{A_{0}^{R}}, \frac{\left(A_{0}^{D}\right)^{2}}{A_{0}^{R}}, \frac{\left(C_{0}^{D}\right)^{2}}{C_{0}^{R}}\right]
$$

- Degenerate case: with

$$
A_{i} \gg B_{i} \gg C_{i}, i=R, D
$$

we get a quasi degenerate mass spectrum

$$
\left[m_{1}, m_{2}, m_{3}\right] \simeq \frac{v^{2}}{\Lambda_{R}}\left[\frac{\left(A_{0}^{D}\right)^{2}}{A_{0}^{R}}, \frac{\left(A_{0}^{D}\right)^{2}}{A_{0}^{R}}, \frac{\left(A_{0}^{D}\right)^{2}}{A_{0}^{R}}\right]
$$

The RH neutrino mass term violates lepton number by two units, and could be a source of lepton asymmetry. The produced asymmetry due to the out of equilibrium decay of the lightest RH neutrino to SM particles is given by [18]:

$$
\epsilon \simeq \frac{3}{16 \pi v^{2}} \frac{1}{\left(\tilde{M}_{\nu}^{D \dagger} \tilde{M}_{\nu}^{D}\right)_{11}} \sum_{j=2,3} \operatorname{Im}\left\{\left[\left(\tilde{M}_{\nu}^{D \dagger} \tilde{M}_{\nu}^{D}\right)_{1 j}\right]^{2}\right\} \frac{M_{R 1}}{M_{R j}}
$$

where $M_{R i}, i=1 \cdots 3$ are the masses for RH neutrinos, and $\tilde{M}_{\nu}^{D}$ is the Dirac neutrino mass matrix in the basis where the Majorana RH neutrino mass matrix $M_{R 0}$ is diagonal. Since the RH neutrino mass matrix (Eq. 65) has the NTB modified tripartite form, then it is diagonalized by $V_{0}^{N T B}$ (Eq. 41). Thus under $\nu_{R} \rightarrow V_{0}^{N T B} \nu_{R}$ we have $M_{\nu 0}^{D} \rightarrow M_{\nu 0}^{D} V_{0}^{N T B}$. We still have freedom in multiplying the diagonalizing unitary matrix $V_{0}^{N T B}$ by diagonal phases $F_{0}=\operatorname{diag}\left(e^{-i \alpha_{1}}, e^{-i \alpha_{2}}, e^{-i \alpha_{3}}\right)$ adjusted normally so that to cancel the phases of the spectrum of $M_{R 0}$. Namely, these phases cancel out upon choosing

$$
\left(\alpha_{1}, \alpha_{2}, \alpha_{3}\right)=\frac{1}{2} \operatorname{Arg}\left(A_{0}^{R}-\frac{49}{47} B_{0}^{R}+\frac{279}{47} C_{0}^{R}, A_{0}^{R}-\frac{49}{47} B_{0}^{R}-\frac{426}{47} C_{0}^{R}, A_{0}^{R}+\frac{51}{47} B_{0}^{R}+\frac{194}{47} C_{0}^{R}\right)(76)
$$

Thus, we have $\tilde{M}_{\nu 0}^{D}=M_{\nu 0}^{D} \cdot V_{0}^{N T B} \cdot F_{0}$ so that we can write

$$
\tilde{M}_{\nu 0}^{D \dagger} \tilde{M}_{\nu 0}^{D}=F_{0}^{\dagger} V_{0}^{N T B \dagger} M_{\nu 0}^{D \dagger} V_{0}^{N T B} V_{0}^{N T B^{\dagger}} M_{\nu 0}^{D} V_{0}^{N T B} F_{0}
$$

which shows that $\tilde{M}_{\nu 0}^{D \dagger} \tilde{M}_{\nu 0}^{D}$ is diagonal and real. Whence $\epsilon$ vanishes in this scenario if S-symmetry is exact. 


\section{The NTB neutrino mass matrix and type-II seesaw scenario}

We apply in this section the type-II seesaw scenario aiming to show that it can solely accommodate an enough lepto/baryogenesis for the observed baryon/photon density in the universe. As in [7], we introduce in this scenario two SM triplet fields $\Sigma_{A}, A=1,2$ which are singlet under the flavor S-symmetry. The Lagrangian part relevant for the neutrino mass matrix is:

$$
\mathcal{L}=\lambda_{\alpha \beta}^{A} L_{\alpha}^{T} C \Sigma_{A} i \tau_{2} L_{\beta}+\mathcal{L}\left(H, \Sigma_{A}\right)+\text { h.c. }
$$

where $A=1,2$ and

$$
\begin{aligned}
\mathcal{L}\left(H, \Sigma_{A}\right)= & \mu_{H}^{2} H^{\dagger} H+\frac{\lambda_{H}}{2}\left(H^{\dagger} H\right)^{2}+M_{A} \operatorname{Tr}\left(\Sigma_{A}^{\dagger} \Sigma_{A}\right)+\frac{\lambda_{\Sigma_{A}}}{2}\left[\operatorname{Tr}\left(\Sigma_{A}^{\dagger} \Sigma_{A}\right)\right]^{2}+ \\
& \lambda_{H \Sigma_{A}}\left(H^{\dagger} H\right) \operatorname{Tr}\left(\Sigma_{A}^{\dagger} \Sigma_{A}\right)+\mu_{A} H^{T} \Sigma_{A}^{\dagger} i \tau_{2} H+h . c .
\end{aligned}
$$

where $H$ and $\Sigma_{A}$ are written as

$$
H=\left(\begin{array}{c}
\phi^{+} \\
\phi^{0}
\end{array}\right), \quad \Sigma_{A}=\left(\begin{array}{cc}
\frac{\Sigma^{+}}{\sqrt{2}} & \Sigma^{0} \\
\Sigma^{++} & -\frac{\Sigma^{+}}{\sqrt{2}}
\end{array}\right)_{A} .
$$

The neutrino mass matrix due to the exchange of the two triplets, $\Sigma_{1}$ and $\Sigma_{2}$, is

$$
\left(M_{\nu}\right)_{\alpha \beta} \simeq v^{2}\left[\lambda_{\alpha \beta}^{1} \frac{\mu_{1}}{M_{\Sigma_{1}}^{2}}+\lambda_{\alpha \beta}^{2} \frac{\mu_{2}}{M_{\Sigma_{2}}^{2}}\right]
$$

where $M_{\Sigma_{i}}$ is the mass of the neutral component $\Sigma_{i}^{0}$ of the triplet $\Sigma_{i}, i=1,2$.

Appropriately, we present some remarks here. First, the flavor S-symmetry would force the matrices $\lambda^{1}$ and $\lambda^{2}$ to have the same NTB modified tripartite structure:

$$
\lambda_{0}^{A}=\left(\begin{array}{ccc}
A_{0}^{A}-B_{0}^{A}+C_{0}^{A} & \frac{7 \sqrt{2}}{47}\left(B_{0}^{A}-22 C_{0}^{A}\right) & \frac{7 \sqrt{2}}{47}\left(B_{0}^{A}+25 C_{0}^{A}\right) \\
\frac{7 \sqrt{2}}{47}\left(B_{0}^{A}-22 C_{0}^{A}\right) & A_{0}^{A}+C_{0}^{A} & \frac{1}{47}\left(49 B_{0}^{A}+191 C_{0}^{A}\right) \\
\frac{7 \sqrt{2}}{47}\left(B_{0}^{A}+25 C_{0}^{A}\right) & \frac{1}{47}\left(49 B_{0}^{A}+191 C_{0}^{A}\right) & A_{0}^{A}-C_{0}^{A}
\end{array}\right), A=1,2
$$

Thus, the neutrino mass matrix in Eq. (81) has the NTB modified tripartite form, hence it can generate all types of neutrino mass hierarchies (Eqs. 36, 38, 40).

Second, the $\mu_{A}$-term in $\mathcal{L}\left(H, \Sigma_{A}\right)$, which does not allow an 'undesirable' spontaneous breaking of the lepton number, permits to arrange the parameters so that minimizing the potential gives a non-zero vev for the neutral component $\Sigma^{0}$ of the triplet. This would generate the mass term for the neutrinos in Eq. (81) in an equivalent way to integrating out the heavy triplets. Third, the flavor changing neutral current due to the triplet is highly suppressed because of the large value of its mass scale (or equivalently due to the smallness of the neutrino masses).

One can discuss now the baryon asymmetry generated by leptogenesis. We show at present that even though the neutrino Yukawa couplings are real it is possible to generate a baryon to photon density consistent with the observations. In fact, since the triplet $\Sigma_{A}$ can decay into lepton pairs $L_{\alpha} L_{\beta}$ and $H H$, it implies that these processes violate total lepton numbers (by two units) and may establish a lepton asymmetry. As the universe cools further, the sphaleron interaction [19] converts this asymmetry into baryon asymmetry. At temperature of the order $\max \left\{M_{1}, M_{2}\right\}$, the heaviest triplet would decay via lepton number violating interactions. Nonetheless, no asymmetry will be generated from this decay since the rapid lepton number violating interactions due to the lightest Higgs triplet will erase any previously generated lepton asymmetry. Therefore, only when the temperature becomes just below the mass of the lightest triplet Higgs the asymmetry would be generated.

With just one triplet, the lepton asymmetry will be generated at the two loop level and it is highly suppressed. We justify this in that one can always redefine the phase of the Higgs field to make the $\mu$ real resulting in the absorptive part of the self energy diagram becoming equal to zero. The choice of 
having more than one Higgs triplet is necessary to generate the asymmetry [20]. In this case, the CP asymmetry in the decay of the lightest Higgs triplet (which we choose to be $\Sigma_{1}$ ) is generated at one loop level due to the interference between the tree and the one loop self energy diagram, and is given by

$$
\epsilon_{C P} \approx-\frac{1}{8 \pi^{2}} \frac{\operatorname{Im}\left[\mu_{1} \mu_{2}^{*} \operatorname{Tr}\left(\lambda^{1} \lambda^{2 \dagger}\right)\right]}{M_{2}^{2}} \frac{M_{1}}{\Gamma_{1}}
$$

where $\Gamma_{1}$ is the decay rate of the lightest Higgs triplet and it is given by

$$
\Gamma_{1}=\frac{M_{1}}{8 \pi}\left[\operatorname{Tr}\left(\lambda^{1 \dagger} \lambda^{1}\right)+\frac{\mu_{1}^{2}}{M_{1}^{2}}\right]
$$

The baryon to photon density is approximately given by

$$
\eta_{B} \equiv \frac{n_{B}}{s}=\frac{1}{3} \eta_{L} \simeq \frac{1}{3} \frac{1}{g_{*} K} \epsilon_{C P}
$$

where $g_{*} \sim 100$ is the number of relativistic degrees of freedom at the time when the Higgs triplet decouples from the thermal bath and $K$ is the efficiency factor which takes into account the fraction of out-of equilibrium decays and the washout effect. In the case of strong wash out, the efficiency factor can be approximated by ( $H$ is the Hubble parameter)

$$
K \simeq \frac{\Gamma_{1}}{H}\left(T=M_{1}\right),
$$

For $\left|\mu_{1,2}\right| \approx M_{\Sigma_{1,2}} \sim 10^{12} \mathrm{GeV}$, and an efficiency factor of order $K \sim 10^{4}$, and assuming real matrices $\lambda^{A}$, and denoting the phases of $\mu_{A}$ by $\phi_{A}$ we find

$$
\eta_{B} \approx 10^{-7} \frac{\operatorname{Tr}\left(\lambda^{1} \lambda^{2 \dagger}\right)}{\operatorname{Tr}\left(\lambda^{1 \dagger} \lambda^{1}\right)+1} \sin \left(\phi_{2}-\phi_{1}\right)
$$

Thus one can produce the correct baryon-to photon ratio of $\eta_{B} \simeq 10^{-10}$ by choosing $\lambda$ 's of order 0.1 and not too small relative phase between the $\mu$ 's.

\section{Summary and conclusion}

We derived an explicit realization of the $\left(Z_{2}\right)^{3}$ symmetry characterizing uniquely the non-tri-bimaximal pattern with $\theta_{z} \neq 0$, in line with the recent neutrino oscillations data. This would constitute a natural explanation for the observed neutrino mixing, rather than considering the tri-bimaximal as the zero approximation followed by some kind of perturbation in order to fit the observed mixing. Actually, the recent oscillation data make the perturbative treatment implausible since large deviations are needed in order to fit the mixing angle $\theta_{z}$. We have imposed the $\left(Z_{2}\right)^{3}$ symmetry in a setup including charged leptons, neutrinos and extra scalar fields in order to account for the charged lepton mass hierarchy. In type-I seesaw scenario, we could account for the various neutrino mass hierarchies. Finally, in type-II seesaw scenario, we could by choosing appropriate Yukawa couplings interpret the observed baryon to photon ratio observed in the universe.

\section{Acknowledgements}

N.C. acknowledges funding provided by the Alexander von Humboldt Foundation. Part of the work was done within the associate scheme program of ICTP. 


\section{References}

[1] K. Nakamura and S. T. Petcov, "Neutrino mass, mixing and oscillation" in K. Nakamura et al. (Particle Data Group), J. Phys. G 37, 075021 (2010).

[2] G. L. Fogli et al., Phys. Rev. D84, 053007 (2011).

[3] Z.Z. Xing; Phys. Lett. B 530 (2002), 159-166.

[4] E. Lashin and N. Chamoun, Phys. Rev. D 85 (2012) 113011.

[5] P.F. Harrison, D.H. Perkins, and W.G. Scott, Phys. Lett. B 530 (2002) 167; P. F. Harrison and W. G. Scott, Phys. Lett. B 535, 163 (2002), Z. z. Xing, Phys. Lett. B 533, 85 (2002), X. G. He and A. Zee, Phys. Lett. B 560, 87 (2003).

[6] E. Ma, Phys. Lett. B 583 (2004) 175.

[7] E. Lashin, E. Malkawi, S. Nasri and N. Chamoun, Phys. Rev. D 80 (2009) 115013.

[8] E. Lashin, E. Malkawi, S. Nasri and N. Chamoun, Phys. Rev. D 83 (2011) 013002.

[9] E. Ma, Phys. Rev. Lett. 90, 221802 (2003).

[10] S.-F. Ge, D. A. Dicus and W. W. Repko, Phys.Lett. B 702 (2011), 220-223; Phys. Rev. Lett. 108 (2012), 041801

[11] P.H. Frampton, S.T. Petcov and W. Rodejohann, Nucl.Phys. B 687 (2004) 31-54; Guido Altarelli, Ferruccio Feruglio, Yin Lin, Nucl.Phys. B 775 (2007) 31-44; I. de Medeiros Varzielas and G. G. Ross, Nucl. Phys. B 733 (2006) 31; C. Luhn, S. Nasri and P. Ramond, Phys. Lett. B 652 (2007) 27; R. N. Mohapatra, S. Nasri and H. B. Yu, Phys. Lett. B 639 (2006) 318; Z. z. Xing, Phys. Lett. B 618 (2005) 141.

[12] Y. Abe et al. [DOUBLE-CHOOZ Collaboration], Phys. Rev. Lett. 108, 131801 (2012) [arXiv:1112.6353 [hep-ex]].

[13] F. P. An et al. [DAYA-BAY Collaboration], Phys. Rev. Lett. 108, 171803 (2012) [arXiv:1203.1669 [hep-ex]].

[14] J. K. Ahn et al. [RENO Collaboration], Phys. Rev. Lett. 108, 191802 (2012) [arXiv:1204.0626 [hep$\mathrm{ex}]$.

[15] D. V. Forero, M. Tortola and J. W. F. Valle, arXiv:1205.4018 [hep-ph].

[16] G. L. Fogli, E. Lisi, A. Marrone and A. Palazzo, Prog. Part. Nucl. Phys. 57, 742 (2006).

[17] J. D. Bjorken and S. Weinberg, Phys. Rev. Lett. 38, 622 (1977).

[18] M. Fukugita and T. Yanagida, Phys. Lett. B 174 (1986) 45.

[19] V. A. Kuzmin, V. A. Rubakov and M. E. Shaposhnikov, Phys. Lett. B 155, 36 (1985).

[20] E. Ma and U. Sarkar, Phys. Rev. Lett. 80, 5716 (1998); T. Hambye, E. Ma and U. Sarkar, Nucl. Phys. B 602, 23 (2001) 